\title{
STRUCTURE-ACTIVITY RELATIONSHIP STUDY OF DITERPENES FOR TREATMENT OF ALZHEIMER'S DISEASE
}

Gabriel F. dos Santos ${ }^{\mathrm{a}}$, Rondinelle G. Pereira ${ }^{\mathrm{a}}$, Maria A. D. Boaventura ${ }^{\mathrm{a}}$, Francisco A. Macias ${ }^{\mathrm{b}}$, Gesiane da S. Lima ${ }^{\mathrm{a}}$, Amanda C. S. Coelho ${ }^{a}$, Jose M. G. Molinillob, Antonio Cala ${ }^{b}$, and Jacqueline A. Takahashia,*

${ }^{a}$ Department of Chemistry, Universidade Federal de Minas Gerais, Av. Antonio Carlos, 31270-901 Belo Horizonte - MG, Brasil bDepartment of Organic Chemistry, Institute of Biomolecules, Campus CEIA3, School of Science, University of Cadiz, C/ Republica Saharaui, 7, 11510-Puerto Real (Cádiz), Spain

Recebido em 15/05/2017; aceito em 19/07/2017; publicado na web em 29/08/2017

\begin{abstract}
Alzheimer's disease is an irreversible, degenerative and age-related disease which is growing more and more with the increase in life expectancy. Kaurane diterpenes are a class of natural products available in large amounts in nature and isolated from plants grown worldwide. In the present work, twenty-seven kaurane diterpenes of natural origin and some readily available derivatives were assayed for acetylcholinesterase inhibition and the structure-activity relationship was analyzed. The kaurenoic acid derivatives screened showed to be promising inhibitors of AChE, which could provide new leads for drugs to fight Alzheimer's disease symptoms. Among them, eleven compounds showed activities comparable or higher than the positive control galantamine. Existence of an allylic hydroxyl group showed to be an important structural feature for AChE inhibition. In addition, presence of free hydroxyl groups at C-17 and C-19, furnished a diol especially active, able to completely inhibit AChE.
\end{abstract}

Keywords: kaurane diterpenes; acetylcholinesterase inhibitors; Alzheimer's disease.

\section{INTRODUCTION}

Alzheimer's disease is among the most disturbing current diseases. It is growing more and more with the increase in life expectancy, being irreversible, degenerative and age-related. This disease is characterized by accumulation of senile plaques, which has beta-amyloid as its major constituent. In Alzheimer's disease, there is huge decline on acetylcholine neurotransmitter level in brain, a fact which is intimately related to memory loss. ${ }^{1-3}$ Numerous approaches have been explored to restore central cholinergic function by means of acetylcholinesterase (AChE) inhibitors since this enzyme is the main protein responsible by acetylcholine hydrolysis. ${ }^{4}$

Natural products have been rediscovered as source of new drugs, due to the success of some secondary metabolites as unique and effective medicines. ${ }^{5}$ Several compounds of natural origin have been described as AChE inhibitors. ${ }^{3,6,7}$ However, many of the natural products able to inhibit AChE activity have been isolated in poor yields from the natural sources, usually plant or microbe extracts. The low availability of such active compounds or their narrow global distribution refrain further drug development and induces the search for active natural compounds available in high yields from readily accessible natural sources.

ent-Kaurenoic acid (1) is a natural product that has been isolated from many plant species such as Sphagneticola trilobata (Asteraceae) ${ }^{8}$ Xylopia sericea,${ }^{9}$ and $X$. frutescens,${ }^{10}$ among other. This compound is usually isolated in high yields, being even possible its straightforward precipitation from crude extracts. Additionally, kaurenoic acid (1) presents many biological activities. ${ }^{11}$ There are some evidences in the literature that kaurane diterpenes can be further studied as new drug candidates to fight Alzheimer's disease. For example, Kim et al reported the neuroprotective effects of a functionalized seco-ent-kauranolide (CBNU06), isolated from Isodon japonicus, which protected PC12 cells against a beta-induced neurotoxicity by inhibiting NF-kappaB signaling pathways, with possible beneficial

*e-mail: jat@qui.ufmg.br effects in Alzheimer's disease. ${ }^{12}$ These features make kaurenoic acid a good model for the development of new acetylcholinesterase inhibitors.

In the present work, twenty-seven kaurane diterpenes of natural origin and some readily available semi-synthetic derivatives were assayed for acetylcholinesterase inhibition and the structure-activity relationship was analyzed.

\section{EXPERIMENTAL}

\section{Natural kaurane diterpenes}

Kaurenoic acid (1) was previously isolated from ethanol extract of Sphagneticola trilobata (Asteraceae) with $9.8 \%$ yield, ${ }^{8}$ and from Xylopia sericea (1.06\% yield). ${ }^{9}$ Compounds $\mathbf{4}$, and $\mathbf{8}$ were also isolated from $X$. sericea with 0.40 and $0.014 \%$ yield, respectively. ${ }^{9} X$. frutescens furnished $\mathbf{1}$ and $\mathbf{4}$ with 4.06 and $3.19 \%$ yield, ${ }^{10}$ respectively. Gibberellic acid (22) was supplied by Across Organics (Hampton, New Hampshire). Stevioside (27) was obtained from Stevita Cristal® (gift of Steviafarma Industrial S.A., Brazil). ${ }^{13}$ Nuclear Magnetic Resonance (NMR) spectra were determined on a Bruker Avance DRX 200 or $400 \mathrm{MHz}$ or on a Agilent $400 \mathrm{MHz}$ with tetramethylsilane (TMS) as internal standard in $\mathrm{CDCl}_{3}, \mathrm{CD}_{3} \mathrm{OD}, \mathrm{CD}_{3} \mathrm{COCD}_{3}$ and $\mathrm{D}_{2} \mathrm{O}$. Chemical shifts $(\delta)$ are given in parts per million (ppm) relative to TMS. Coupling constants $(J)$ are given in hertz $(\mathrm{Hz})$.

\section{Semisynthesis of derivatives}

Treatment of $\mathbf{1}$ and $\mathbf{4}$ with iodomethane ${ }^{14}$ gave the respective methyl esters $\mathbf{2}$ and $\mathbf{6}$, with nearly quantitative yields. Reduction of $\mathbf{2}$ from $\mathrm{LiAlH}_{4}$ gave the alcohol derivative $\mathbf{3}$ ( $84 \%$ yield). ${ }^{15}$ Hydrolysis of $\mathbf{4}$ from $\mathrm{KOH} 1 \%$ in methanol for $5 \mathrm{~h}$ gave the alcohol derivative $\mathbf{5}$ (93\% yield) $;{ }^{15}$ this later, was treated with iodomethane to give $7^{15}(98 \%$ yield). Monoamides 9-12, 14 and 15 and diamide 16 were obtained from reaction between $\mathbf{1}$ and the corresponding amines, according to methodology adapted from Bandgar and Bettigeri (2004) with 
temperature at $\sim-20{ }^{\circ} \mathrm{C}$ for $10 \mathrm{~min} .{ }^{16}$ After purification by column chromatography, the monoamides were obtained in a $41-70 \%$ yield range. The diamide $\mathbf{1 6}$ was isolated as a reaction side product with $27 \%$ yield. Compound 17 was synthesized by catalytic hydrogenation of kaurenoic acid (1), according to Prakash and Prakash (2013) ${ }^{17}$ (71\% yield). Monoamine 18 was obtained from reaction between 17 and the corresponding amine using the same methodology used for preparation of amides 9-12, with 39\% yield. Compound 19 and $\mathbf{2 1}$ were prepared by treatment of $\mathbf{2}$ and $\mathbf{3}$ with diborane generated in situ, with $\mathrm{NaBH}_{4}$ and $\mathrm{BF}_{3} \mathrm{OEt}_{2},{ }^{18}$ with 86 and $93 \%$ yield, respectively.

Compounds 13, 20 and 23 were obtained by a $\mathrm{SN}_{2}$-type reaction between 1, 19 and gibberelic acid (22), respectively, and the bromolactone 5-bromo-3-methyl $2(5 \mathrm{H})$-furanone. ${ }^{19}$ After purification by column chromatography, compounds $\mathbf{1 3 , 2 0}$ and 23 were obtained with 75, 14 and $62 \%$ yield, respectively. Compounds $\mathbf{2 4}$ and $\mathbf{2 5}$ were prepared from $\mathrm{GA}_{3}(\mathbf{2 2})$ and benzyl bromide, according to Chen et al. ${ }^{20}$ with 74 and $22.5 \%$ yield, respectively. Compound 26 was obtained by reaction of $\mathbf{2 4}$ with acetic anhydride in pyridine at 0 ${ }^{\circ} \mathrm{C}^{21}$ with $85 \%$ yield.

\section{Assay for AChE Inhibitory Activity}

The assays for all compounds were performed in 96 well microplates using the spectrophotometric method according to Ellman et al. ${ }^{22}$ and modifications. ${ }^{23}$ Acetylthiocholine iodide $\left(15 \mathrm{mmol} \mathrm{L}^{-1}\right)$ ( $25 \mu \mathrm{L}$ ) were added to the wells, together with $125 \mu \mathrm{L}$ of Ellman's reagent, 5,5'-dithio-bis-(2-nitrobenzoic acid) (DTNB) $\left(3 \mathrm{mmol} \mathrm{L}^{-1}\right)$ containing $0.1 \mathrm{~mol} \mathrm{~L}^{-1} \mathrm{NaCl}$ and $0.02 \mathrm{~mol} \mathrm{~L}^{-1} \mathrm{MgCl}_{2} \cdot 6 \mathrm{H}_{2} \mathrm{O}, 50 \mu \mathrm{L}$ of Tris/ $\mathrm{HCl}\left(50 \mathrm{mmol} \mathrm{L}^{-1}\right) \mathrm{pH} 8$ and $25 \mu \mathrm{L}$ of sample solution (DMSO $10 \mathrm{mg} / \mathrm{mL}$ ). To determine AChE inhibition extention, plates were read in a micro plate reader and absorbance was measured at every 60 seconds for eight times totaling eight minutes of reading. After this stage, there were added $25 \mu \mathrm{L}$ of AChE solution $(0.222 \mathrm{U} / \mathrm{mL})$ containing $0.1 \%$ bovine serum albumin to the wells. A new reading was held 2 minutes AChE addition after and the absorbance measured immediately and then at every 60 seconds for nine times, totaling nine minutes of reading. Galantamine was used as a positive control. Assay was carried out in quintuplicate and absorbance was measured at 405 $\mathrm{nm}$ in a microplate reader Biotec ELX 800. AChE percent inhibition was calculated using the following equation:

Inhibition $(\%)=100-\left[\left(\mathrm{R}_{\text {sample }} / \mathrm{R}_{\text {control }}\right) * 100\right]$, where $\mathrm{R}_{\text {sample }}=$ rate of sample extracts reaction and $\mathrm{R}_{\text {control }}=$ rate of blank.

\section{RESULTS AND DISCUSSION}

The evaluation of antiacetylcholinesterase activity bioassay was realized with five natural products $(\mathbf{1}, \mathbf{4}, \mathbf{8}, 22$ and 27$)$ and twenty two natural product derivatives $(\mathbf{2}, \mathbf{3}, \mathbf{5}-\mathbf{7}, \mathbf{9}-\mathbf{2 1}, \mathbf{2 3}-\mathbf{2 6})$. The chemical structures of these compounds are presented in Figure 1.

All compounds (1-27) were fully characterized by ${ }^{1} \mathrm{H}$ and ${ }^{13} \mathrm{C}$ NMR in comparison to data reported in the literature. Their ${ }^{13} \mathrm{C} N M R$ data are shown in Tables 1-3.

Natural compounds $1,4,8,22$ and 27 were initially screened for their capacity for $\mathrm{AChE}$ inhibition. Among them, the most active ones were $\mathbf{1}$ (kaurenoic acid) $(81.6 \pm 1.8 \%)$ and 22 (gibberellic acid) $(85.2 \pm 4.1)$. Presence of a bulk sugar moiety at C-13 and C-19 did not have the same effect in the activity, since compound 27 was not very active $(36.7 \pm 4.3 \%)$. These results have encouraged us to prepare derivatives from them, as well as other derivatives with kaurane skeleton, such as the respective methyl esters and some kaurane derivatives functionalized at C-15, C-16 and C-19.

Regarding to kaurenoic acid derivatives, natural product $\mathbf{1}$ was more active than its methyl ester derivative $2(74.2 \pm 3.1 \%)$. When the carboxyl group was replaced by an alcohol at C-19 in 3, activity (79.3 $\pm 4.1 \%$ ) was not different from that of compound 1 . There was also no significant difference between the activity of kaurenoic acid (1) $(81.6 \pm 1.8)$ and derivative $\mathbf{1 7}(82.0 \pm 1.6)$. The presence of an allylic hydroxyl group at C-15 (compound 5) led to one of the most active derivatives which was able to inhibit AChE in a higher level (93.0 \pm $3.8 \%)$ compared to galantamine $(90.3 \pm 0.5)$, the control used in the experiments. It is very interesting to observe that galantamine also has an allylic hydroxyl group on its structure (Figure 2). This hydroxyl group acts as a donor in the bonding with Glu202 and, therefore, is involved in the biological activity mechanism. ${ }^{26}$ Other galantamine derivatives bearing this allylic hydroxyl group were also reported as potent AChE inhibitors. ${ }^{27}$

The presence of the acetoxy group at C-15 in $\mathbf{4}$ may be responsible for decreasing the activity $(65.0 \pm 4.5 \%)$ of this natural product when compared to $1(81.6 \pm 1.8)$. It is worth mentioning that the biological activity of compound $6(54.6 \pm 4.0 \%)$, derived from 4 by esterification at C-19, was even lower. However, this is not the unique structural feature important for biological response, since compound 7, also bearing an allylic hydroxyl group, was much less active (59.0 \pm 3.7 ) than kaurenoic acid (1) probably due to the absence of a free carboxyl group at C-19.

Insertion of a monoamide group at $\mathrm{C}-19$ in kaurenoic acid greatly decreased the activity and this activity decreased with the size of the side chain, as derivatives $\mathbf{9 ,} \mathbf{1 0}$ and $\mathbf{1 1}$ were poorly active.

However, reduction of activity was not observed by introduction of the monoamine group at $\mathrm{C}-19$ of $\mathbf{1 7}(82.0 \pm 1.6 \%)$ to yield compound $18(89.8 \pm 0.5 \%)$. Cyclic monoamide $12(62.9 \pm 2.3)$ inhibited acetylcholinesterase much more than the corresponding linear amide (derivatives 9-11). Concerning to amides with side chains of the same size, diamide $\mathbf{1 6}(81.8 \pm 1.0 \%)$ was the most active than derivative $9(34.1 \pm 2.0 \%)$.

Monoamides synthesized from aromatic diamines (compounds 14 and 15) were more active than those derived from aliphatic diamines, leading into consideration the distance between the two nitrogen atoms (compounds $\mathbf{1 1}$ and 15). It is noteworthy that non substituted aromatic amide $\mathbf{1 4}$ was more active $(56.4 \pm 4.2)$ than the corresponding $p$-amino substituted one (39.3 \pm 4.9$)$. Introduction of a butenolide substituent promoted different effects in the activity. When added to ring $\mathrm{D}$ (compound 20), the activity was very weak $(16.3 \pm 0.5)$, while, when bonded to the C-19 carbonyl (compound 13), the activity has greatly increased $(81.8 \pm 1.0)$. The most active derivative from kaurenoic acid series was the diol 21, which was able to completely inhibit AChE activity (100\%) at the conditions used, being more active than the positive control.

Gibberellic acid (22) is a hormone biosynthesized from kaurenoic acid in the plants. Its activity was comparable $(85.2 \pm 4.1)$ to that of kaurenoic acid (1) motivating the screening of a series of compounds prepared from 22 (compounds 23-26). Derivative 25 showed the same level of AChE inhibition than the starting material 22, while introduction of a butenolide substituent in gibberellic acid structure (derivative 23) decreased the activity $(68.3 \pm 3.8)$.

Based on the obtained results, the kaurenoic and gibberellic acids derivatives showed to be promising inhibitors of AChE, which could provide new leads for development of drugs for treating Alzheimer's disease. Among them, compounds 1, 5, 13, 16-18, 21 , 22 and 25 showed relevant activities (higher than 80\%), and among them, compounds 5, 18 and $\mathbf{2 1}$ presented activities comparable to the positive control, galanthamine. Particularly, compound 21 which bears two free hydroxyl groups at C-17 and C-19, was especially active, completely inhibiting the AChE (100\%). Results for AChE inhibition obtained for all compounds are presented in Table 4. 

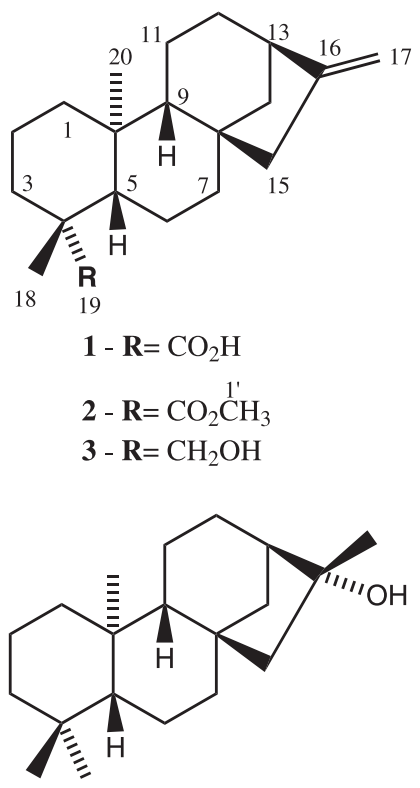

8

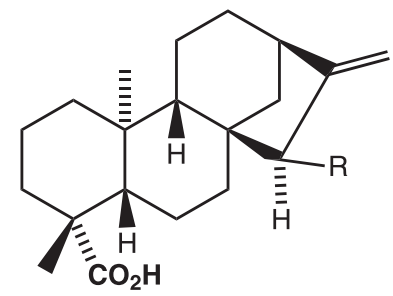

$4-\mathbf{R}=\stackrel{1^{\prime}}{\mathrm{OCOC}^{\prime}} \mathrm{L}_{3}$

$\mathbf{5}-\mathbf{R}=\mathrm{OH}$

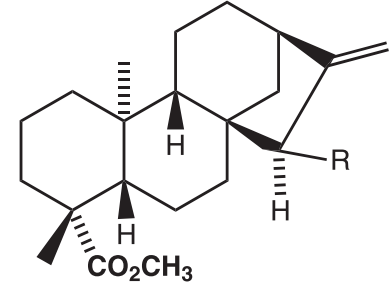

$\mathbf{6}-\mathbf{R}=\stackrel{1^{\prime}}{\mathrm{OCOC}^{\prime}} \mathrm{H}_{3}$

$\mathbf{7}-\mathbf{R}=\mathrm{OH}$

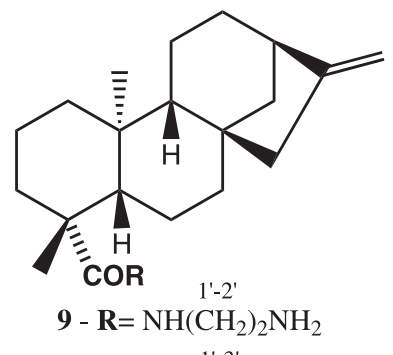

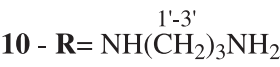

$11-\mathbf{R}=\mathrm{NH}\left(\mathrm{CH}_{2}-\mathrm{CH}_{2}\right)_{4} \mathrm{NH}_{2}$

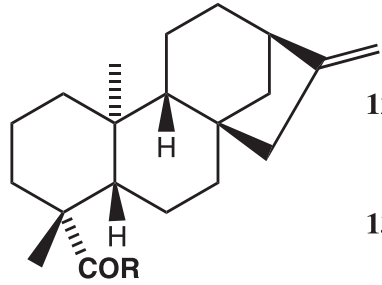

$12-\mathbf{R}=$<smiles>C1CNCCN1</smiles>

$13-\mathbf{R}=0 \underbrace{1^{\prime}, \psi^{4}}_{2^{\prime}}$

$\mathbf{1 4}-\mathbf{R}=$ NHAr

$15-\mathbf{R}=p-\mathrm{NHArNH}_{2}$
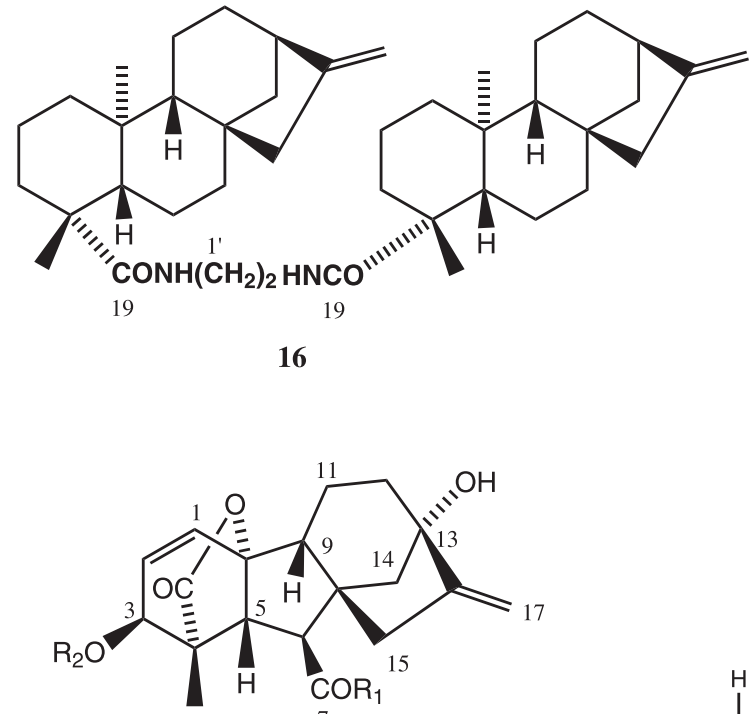

$22-\mathbf{R}_{\mathbf{1}}=\mathrm{OH} \quad{ }^{7} \mathbf{R}_{\mathbf{2}}=\mathrm{H}$

$23-\mathbf{R}_{\mathbf{1}}=$ O $\underbrace{1^{\prime} \mathrm{O}}_{2^{\prime}} \underbrace{4^{\prime}}_{5^{\prime}} \mathbf{R}_{\mathbf{2}}=\mathrm{H}$

$24-\mathbf{R}_{\mathbf{1}}=\stackrel{\mathrm{OCH}^{\prime}}{\mathrm{O}_{2} \mathrm{~A}^{\prime} 5^{\prime}} \quad \mathbf{R}_{\mathbf{2}}=\mathrm{H}$

$25-\mathbf{R}_{\mathbf{1}}=\mathrm{OCH}_{2} \mathrm{Ar} \quad \mathbf{R}_{\mathbf{2}}=\stackrel{6^{\prime}}{\mathrm{CH}_{2} \mathrm{Ar}}$

$26-\mathbf{R}_{\mathbf{1}}=\mathrm{OCH}_{2} \mathrm{Ar} \quad \mathbf{R}_{\mathbf{2}}=\underset{6^{\prime}}{\mathrm{COCH}_{3}}$

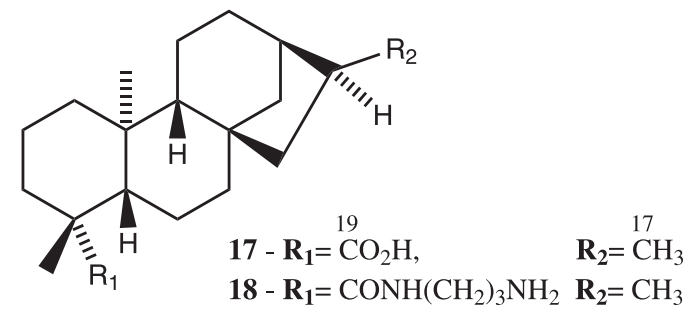

$19-\mathbf{R}_{\mathbf{1}}=\mathrm{CO}_{2}{ }^{\prime} \mathrm{CH}_{3}$ $\mathbf{R}_{\mathbf{2}}=\mathrm{CH}_{2} \mathrm{OH}$

20 - $\mathbf{R}_{\mathbf{1}}=\mathrm{CO}_{2} \stackrel{1^{\prime}}{\mathrm{C}} \mathrm{H}_{3}$

$21-\mathbf{R}_{\mathbf{1}}=\mathrm{CH}_{2} \mathrm{OH}$ $\mathbf{R}_{\mathbf{2}}=0 \overbrace{3^{\prime}}^{2^{\prime}}$ $\mathbf{R}_{\mathbf{2}}=\mathrm{CH}_{2} \mathrm{OH}$

Figure 1. Chemical structures of the kaurane diterpenes tested

\section{ACNOWLEDGEMENTS}

The authors are grateful to the Brazilian Funding Agencies Conselho Nacional de Desenvolvimento Científico e Tecnológico (CNPq), Coordenação de Aperfeiçoamento de Ensino Superior
(CAPES) and Fundação de Amparo à Pesquisa do Estado de Minas Gerais (FAPEMIG) (CEX APQ 02604/16) for fellowships and financial support. GSL is a CAPES grantee (BEX6447/15-5). This research was also supported by 'Ministerio de Economía y Competitividad' (MINECO), Spain, Project AGL2013-42238-R. 
Table 1. ${ }^{13} \mathrm{C}$ NMR data obtained for compounds 1-9 $\left(\mathrm{CDCl}_{3}\right)$

\begin{tabular}{|c|c|c|c|c|c|c|c|c|c|}
\hline \multirow{2}{*}{ Carbon } & \multicolumn{9}{|c|}{ Compound } \\
\hline & $\mathbf{1}^{24}$ & $2^{24}$ & $3^{24}$ & $4^{25}$ & $\mathbf{5}^{25}$ & $6^{25}$ & $7^{25}$ & $\mathbf{8}^{25}$ & $\mathbf{9}^{15}$ \\
\hline 1 & 40.7 & 40.8 & 40.8 & 40.7 & 40.6 & 41.0 & 40.6 & 42.1 & 41.9 \\
\hline 2 & 19.1 & 19.2 & 18.3 & 19.1 & 19.1 & 19.3 & 19.1 & 18.6 & 20.7 \\
\hline 3 & 37.7 & 38.2 & 35.8 & 37.7 & 38.8 & 39.0 & 37.9 & 42.1 & 39.0 \\
\hline 4 & 43.8 & 43.9 & 38.7 & 43.7 & 43.7 & 43.9 & 43.8 & 33.2 & 45.1 \\
\hline 5 & 57.0 & 57.1 & 56.9 & 56.5 & 45.4 & 47.2 & 56.4 & 56.2 & 59.1 \\
\hline 6 & 21.8 & 21.9 & 21.6 & 21.3 & 21.5 & 21.5 & 21.5 & 20.4 & 23.6 \\
\hline 7 & 41.3 & 41.3 & 41.4 & 38.8 & 37.9 & 38.2 & 38.9 & 40.3 & 42.9 \\
\hline 8 & 43.7 & 44.2 & 44.2 & 45.9 & 45.6 & 46.1 & 45.7 & 45.3 & 45.6 \\
\hline 9 & 55.1 & 55.1 & 56.3 & 46.9 & 56.3 & 56.8 & 45.5 & 56.8 & 56.7 \\
\hline 10 & 39.6 & 39.4 & 39.3 & 39.4 & 39.0 & 39.3 & 39.1 & 39.3 & 40.9 \\
\hline 11 & 18.4 & 18.4 & 18.3 & 17.9 & 18.2 & 18.1 & 18.3 & 17.9 & 19.6 \\
\hline 12 & 33.1 & 33.1 & 33.3 & 33.3 & 33.0 & 33.3 & 33.1 & 26.9 & 34.4 \\
\hline 13 & 44.2 & 43.8 & 44.0 & 40.6 & 40.0 & 40.8 & 40.0 & 49.0 & 45.4 \\
\hline 14 & 39.7 & 39.7 & 39.7 & 36.4 & 36.2 & 36.5 & 36.3 & 37.7 & 40.9 \\
\hline 15 & 48.9 & 48.9 & 49.3 & 81.6 & 82.4 & 81.8 & 82.5 & 58.0 & 50.2 \\
\hline 16 & 155.9 & 155.9 & 155.6 & 153.7 & 158.4 & 153.9 & 158.4 & 79.3 & 156.9 \\
\hline 17 & 103.0 & 102.9 & 102.9 & 106.1 & 104.7 & 106.1 & 104.7 & 24.4 & 103.9 \\
\hline 18 & 29.0 & 28.9 & 27.1 & 28.9 & 28.7 & 28.7 & 28.7 & 33.5 & 30.5 \\
\hline 19 & 184.6 & 178.1 & 65.6 & 184.2 & 178.0 & 178.0 & 178.1 & 21.5 & 180.5 \\
\hline 20 & 15.6 & 15.4 & 18.1 & 15.8 & 15.4 & 15.7 & 15.5 & 17.7 & 16.8 \\
\hline $1 '$ & & 51.1 & & 171.4 & & 171.3 & 51.1 & & 41.6 \\
\hline $2^{\prime}$ & & & & 21.3 & & 21.2 & & & 41.0 \\
\hline $3^{\prime}$ & & & & & & 50.9 & & & \\
\hline
\end{tabular}

Superscript numbers refer to the literature data.

Table 2. ${ }^{13} \mathrm{C}$ NMR data obtained for compounds 10-18 $\left(\mathrm{CDCl}_{3}\right)$

\begin{tabular}{|c|c|c|c|c|c|c|c|c|c|}
\hline \multirow{2}{*}{ Carbon } & \multicolumn{9}{|c|}{ Compound } \\
\hline & $\mathbf{1 0}^{15}$ & $\mathbf{1 1}^{15}$ & $12^{15}$ & $\mathbf{1 3}^{15}$ & $14^{15}$ & $15^{15}$ & $16^{15}$ & $17^{15}$ & $18^{15}$ \\
\hline 1 & 41.3 & 42.5 & 41.8 & 40.6 & 41.1 & 41.1 & 41.2 & 40.0 & 41.2 \\
\hline 2 & 19.6 & 20.7 & 20.1 & 19.0 & 19.4 & 19.3 & 19.4 & 19.5 & 19.4 \\
\hline 3 & 39.7 & 39.0 & 39.5 & 37.9 & 38.5 & 38.4 & 38.2 & 38.3 & 38.3 \\
\hline 4 & 43.9 & 45.1 & 46.1 & 44.2 & 44.3 & 44.3 & 43.7 & 44.1 & 43.8 \\
\hline 5 & 57.6 & 59.2 & 61.4 & 57.1 & 57.5 & 57.4 & 57.4 & 57.5 & 57.4 \\
\hline 6 & 22.6 & 23.7 & 22.9 & 21.9 & 22.6 & 22.5 & 22.4 & 22.5 & 22.8 \\
\hline 7 & 41.6 & 43.0 & 42.2 & 41.2 & 41.5 & 41.5 & 41.5 & 42.6 & 42.4 \\
\hline 8 & 44.5 & 45.6 & 44.4 & 43.8 & 44.7 & 44.7 & 44.3 & 45.2 & 44.8 \\
\hline 9 & 55.2 & 56.7 & 56.3 & 55.0 & 55.1 & 55.1 & 55.1 & 57.0 & 56.6 \\
\hline 10 & 38.5 & 41.0 & 40.1 & 39.6 & 39.6 & 39.7 & 39.6 & 40.0 & 39.6 \\
\hline 11 & 18.6 & 19.7 & 18.5 & 18.4 & 18.5 & 18.5 & 18.4 & 19.4 & 19.0 \\
\hline 12 & 33.2 & 34.4 & 33.1 & 33.0 & 33.0 & 33.0 & 33.1 & 26.3 & 25.9 \\
\hline 13 & 43.8 & 45.4 & 43.9 & 44.2 & 43.8 & 43.7 & 43.8 & 40.4 & 40.0 \\
\hline 14 & 38.4 & 40.9 & 40.2 & 39.7 & 39.7 & 39.6 & 39.7 & 41.2 & 40.0 \\
\hline 15 & 49.0 & 50.2 & 49.0 & 48.9 & 48.8 & 48.8 & 48.9 & 49.1 & 48.7 \\
\hline 16 & 156.1 & 156.9 & 156.2 & 155.6 & 155.7 & 155.7 & 155.8 & 34.8 & 34.4 \\
\hline 17 & 103.1 & 103.9 & 102.7 & 103.1 & 103.2 & 103.1 & 103.0 & 16.2 & 15.7 \\
\hline 18 & 30.3 & 30.6 & 28.0 & 28.6 & 29.8 & 29.8 & 30.1 & 29.4 & 30.2 \\
\hline 19 & 177.3 & 179.8 & 176.7 & 175.9 & 175.2 & 175.3 & 178.3 & 185.0 & 177.6 \\
\hline 20 & 15.9 & 16.9 & 18.2 & 15.9 & 15.8 & 15.8 & 15.8 & 15.9 & 15.7 \\
\hline $1^{\prime}$ & 38.1 & 40.2 & 47.0 & 92.3 & 138.0 & 139.0 & 40.4 & & 37.0 \\
\hline $2^{\prime}$ & 32.3 & 27.6 & 46.1 & 142.1 & 120.3 & 107.2 & & & 30.4 \\
\hline $3^{\prime}$ & 40.4 & 28.7 & 47.0 & 134.4 & 129.0 & 146.8 & & & 38.7 \\
\hline $4^{\prime}$ & & 41.4 & 46.1 & 171.3 & 124.2 & 110.2 & & & \\
\hline $5^{\prime}$ & & & & 10.7 & 129.0 & 129.7 & & & \\
\hline $6^{\prime}$ & & & & & 120.3 & 111.1 & & & \\
\hline
\end{tabular}

Superscript numbers refer to the literature data. 
Table 3. ${ }^{13} \mathrm{C}$ NMR data obtained for compounds 19-20, 23 and 25-26 $\left(\mathrm{CDCl}_{3}\right), \mathbf{2 1 - 2 2}\left(\mathrm{CD}_{3} \mathrm{OD}\right), 24\left(\mathrm{C}_{3} \mathrm{D}_{6} \mathrm{O}\right)$, and $27\left(\mathrm{D}_{2} \mathrm{O}\right)$

\begin{tabular}{|c|c|c|c|c|c|c|c|c|c|}
\hline \multirow{2}{*}{ Carbon } & \multicolumn{9}{|c|}{ Compound } \\
\hline & $19^{15}$ & $\mathbf{2 0}^{15}$ & $21^{15}$ & $\mathbf{2 2}^{15}$ & $\mathbf{2 3}^{15}$ & $\mathbf{2 4}^{15}$ & $\mathbf{2 5}^{15}$ & $26^{15}$ & $27^{13}$ \\
\hline 1 & 40.7 & 40.7 & 41.6 & 133.1 & 132.7 & 131.9 & 132.5 & 134.2 & 40.7 \\
\hline 2 & 19.1 & 19.1 & 19.1 & 133.8 & 132.5 & 133.1 & 130.4 & 129.1 & 19.0 \\
\hline 3 & 39.4 & 39.4 & 36.5 & 70.3 & 69.6 & 69.3 & 75.8 & 70.2 & 38.1 \\
\hline 4 & 43.6 & 43.8 & 39.6 & 54.8 & 53.4 & 53.4 & 53.6 & 52.1 & 44.1 \\
\hline 5 & 57.0 & 57.0 & 58.1 & 53.8 & 52.8 & 52.7 & 53.4 & 53.4 & 57.2 \\
\hline 6 & 22.2 & 22.2 & 21.5 & 52.2 & 51.2 & 51.1 & 50.9 & 50.6 & 21.8 \\
\hline 7 & 42.1 & 41.9 & 43.6 & 180.8 & 170.6 & 171.6 & 171.6 & 171.6 & 41.5 \\
\hline 8 & 43.8 & 43.8 & 45.2 & 51.0 & 50.2 & 50.3 & 50.4 & 50.4 & 42.5 \\
\hline 9 & 56.4 & 56.2 & 59.0 & 52.0 & 50.6 & 51.1 & 51.1 & 51.1 & 53.6 \\
\hline 10 & 40.3 & 40.0 & 40.2 & 92.3 & 90.3 & 90.2 & 90.5 & 90.0 & 39.6 \\
\hline 11 & 19.1 & 19.1 & 19.8 & 17.8 & 17.0 & 16.8 & 16.9 & 16.9 & 20.4 \\
\hline 12 & 26.0 & 26.1 & 26.7 & 39.6 & 38.0 & 38.9 & 38.1 & 38.1 & 36.1 \\
\hline 13 & 38.1 & 38.1 & 38.4 & 78.4 & 78.0 & 77.2 & 78.1 & 78.1 & 85.8 \\
\hline 14 & 36.9 & 37.2 & 41.3 & 45.3 & 44.8 & 44.7 & 42.9 & 44.8 & 44.1 \\
\hline 15 & 44.2 & 44.2 & 45.0 & 44.0 & 42.7 & 42.9 & 44.9 & 42.8 & 47.2 \\
\hline 16 & 43.2 & 40.3 & 44.0 & 158.1 & 156.3 & 157.8 & 157.1 & 156.8 & 153.8 \\
\hline 17 & 64.3 & 71.9 & 64.0 & 107.3 & 108.2 & 105.9 & 107.3 & 107.5 & 104.2 \\
\hline 18 & 28.7 & 28.7 & 27.6 & 14.8 & 14.6 & 14.1 & 14.6 & 14.3 & 28.1 \\
\hline 19 & 178.1 & 178.1 & 64.9 & 175.0 & 178.1 & 178.1 & 178.5 & 177.1 & 176.8 \\
\hline 20 & 15.3 & 15.3 & 18.7 & & - & - & - & 67.0 & 15.1 \\
\hline $1^{\prime}$ & 51.1 & 51.1 & & & 92.5 & 66.2 & 66.8 & 135.3 & 97.1 \\
\hline $2^{\prime}$ & & 101.8 & & & 151.6 & 136.2 & 135.4 & 128.4 & 84.1 \\
\hline $3^{\prime}$ & & 143.0 & & & 134.9 & 128.3 & 128.5 & 128.6 & 77.7 \\
\hline $4^{\prime}$ & & 134.0 & & & 170.8 & 128.4 & 128.6 & 128.6 & 71.3 \\
\hline 5 & & 172.0 & & & 10.7 & 128.1 & 128.4 & - & 77.9 \\
\hline $6^{\prime}$ & & 10.6 & & & & & 72.9 & 170.0 & 62.6 \\
\hline 1" & & & & & & & 137.6 & 20.8 & 106.1 \\
\hline $2 "$ & & & & & & & 127.9 & & 75.9 \\
\hline 3" & & & & & & & 128.4 & & 76.9 \\
\hline $4 "$ & & & & & & & 128.0 & & 71.9 \\
\hline $5 "$ & & & & & & & & & 77.0 \\
\hline 6" & & & & & & & & & 62.8 \\
\hline $1 "$, & & & & & & & & & 94.9 \\
\hline $2 "$, & & & & & & & & & 73.2 \\
\hline $3 "$, & & & & & & & & & 78.0 \\
\hline $4 "$, & & & & & & & & & 70.4 \\
\hline $5 "$, & & & & & & & & & 78.3 \\
\hline 6"" & & & & & & & & & 61.8 \\
\hline
\end{tabular}

Superscript numbers refer to the literature data.

Table 4. Percentage of inhibition of galantamine and studied diterpenes

\begin{tabular}{cccccc}
\hline Sample & Concentration $\left(\mathrm{mmol} \mathrm{L}^{-1}\right)$ & \% Inhibition $($ mean $\pm \mathrm{sd})$ & Sample & Concentration $\left(\mathrm{mmol} \mathrm{L}^{-1}\right)$ & \% Inhibition $(\mathrm{mean} \pm \mathrm{sd})$ \\
\hline $\mathbf{1}$ & 33.1 & $81.6 \pm 1.8$ & $\mathbf{1 5}$ & 25.5 & $39.3 \pm 4.9$ \\
$\mathbf{2}$ & 31.6 & $74.2 \pm 3.1$ & $\mathbf{1 6}$ & 15.9 & $81.8 \pm 1.0$ \\
$\mathbf{3}$ & 34.7 & $79.3 \pm 4.1$ & $\mathbf{1 7}$ & 32.8 & $82.0 \pm 1.6$ \\
$\mathbf{4}$ & 27.8 & $65.0 \pm 4.5$ & $\mathbf{1 8}$ & 27.8 & $89.8 \pm 0.5$ \\
$\mathbf{5}$ & 31.4 & $93.0 \pm 3.8$ & $\mathbf{1 9}$ & 29.9 & $79.2 \pm 1.3$ \\
$\mathbf{6}$ & 26.7 & $54.6 \pm 4.0$ & $\mathbf{2 0}$ & 23.3 & $16.3 \pm 0.5$ \\
$\mathbf{7}$ & 30.1 & $59.0 \pm 3.7$ & $\mathbf{2 1}$ & 32.7 & $100.0 \pm 3.1$ \\
$\mathbf{8}$ & 34.5 & $34.8 \pm 4.6$ & $\mathbf{2 2}$ & 28.9 & $85.2 \pm 4.1$ \\
$\mathbf{9}$ & 29.1 & $34.1 \pm 2.0$ & $\mathbf{2 3}$ & 22.6 & $78.3 \pm 3.8$ \\
$\mathbf{1 0}$ & 27.9 & $31.5 \pm 1.7$ & $\mathbf{2 4}$ & 22.9 & $84.9 \pm 2.0$ \\
$\mathbf{1 1}$ & 26.9 & $16.2 \pm 1.0$ & $\mathbf{2 5}$ & 19.0 & 7.0 \\
$\mathbf{1 2}$ & 27.0 & $62.9 \pm 2.3$ & $\mathbf{2 6}$ & 20.9 & 3.9 \\
$\mathbf{1 3}$ & 25.1 & $81.8 \pm 1.0$ & $\mathbf{2 7}$ & 12.4 & $36.7 \pm 4.3$ \\
$\mathbf{1 4}$ & 26.5 & $56.4 \pm 4.2$ & Galantamine & 34.8 & $90.3 \pm 0.5$ \\
\hline
\end{tabular}




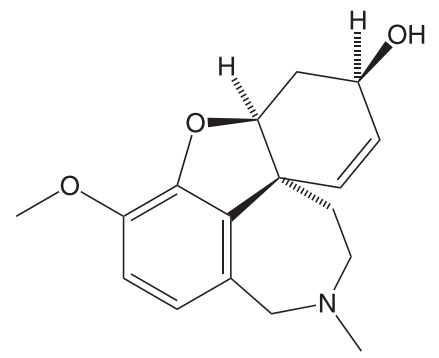

Figure 2. Chemical structure of galantamine, showing the allylic alcohol on a six membered-ring, which has some structural resemblance with the structure of some tested compounds

\section{REFERENCES}

1. Yoo, K.-Y.; Park, S.-Y.; Molecules 2012, 17, 3524.

2. Arunkhamkaew, S.; Athipornchai, A.; Apiratikul, N.; Suksamrarn, A.; Ajavakom, V.; Bioorg. Med. Chem. Lett. 2013, 23, 2880.

3. Cahlíková, L.; Pérez, D. I.; Štěpánková, Š.; Chlebek, J.; Šafratová, M.; Hošt'álková, A.; Opletal, L.; J. Nat. Prod. 2015, 78, 1189.

4. López, M. D.; Campoy, F. J.; Pascual-Villalobos, M. G.; MuñozDelgado, E.; Vidal, C. J.; Chem. Biol. Interact. 2015, 229, 36.

5. Newman, D. J.; Cragg, G. M.; J. Nat. Prod. 2012, 75, 311.

6. Houghton, P. J.; Ren, Y.; Howes, M. -J.; Nat. Prod. Rep. 2006, 23, 181.

7. Zhang, C.; Ji, J.; Ji, M.; Fan, P.; Phytochem. Lett. 2015, 12, 215.

8. Vieira, H. S.; Takahashi, J. A.; Boaventura, M. A. D.; Fitoterapia 2001, 72,854 .

9. Takahashi, J. A.; Vieira, H. S.; Boaventura, M. A. D.; Hanson, J. R.; Hitchcock, P. B.; de Oliveira, A. B.; Quim. Nova 2001, 24, 616.

10. Takahashi, J. A.; Boaventura, M. A. D. ; Oliveira, A. B. ; Bayma, J. C.; J. Nat. Prod. 1995, 40, 607.
11. Ghisalberti E. I.; Fitoterapia 1997, LXVIII, 303.

12. Kim, H. S.; Lim, J. Y.; Sul, D.; Hwang, B. Y.; Won, T. J.; Hwang, K. W.; Park, S. Y.; Eur. J. Pharmacol. 2009, 622, 25.

13. Milagre, H. M. S.; Martins, L. R.; Takahashi, J. A.; Braz. J. Microbiol. 2009, 40, 367.

14. Boeck, P.; Sá, M. M.; Souza, B. S.; Cercená, R.; Escalante, A. M.; Zachino, S. A.; Cechinel Filho, V.; Yunes, R. A.; J. Braz. Chem. Soc. 2005, 16, 1360 .

15. Pereira, R. G.; Tese de Doutorado, Universidade Federal de Minas Gerais, Brasil, 2016

16. Bandgar, R. P.; Bettigeri, S. V.; Synth. Commun. 2004, 34, 2917.

17. Prakash, V. S.; Prakash, I.; Int. J. Mol. Sci. 2013, 14, 15669.

18. Batista, R.; Humberto, J. L.; Chiari, E.; de Oliveira, A. B.; Bioorg. Med. Chem. 2007, 15, 381.

19. Macías, F. A.; Gacía-Díaz, M. D.; Pérez-de-Luque, A.; Rubiales, D.; Galindo, J. C. G.; J. Agric. Food Chem. 2009, 57, 5853.

20. Chen, J.; Sun, Z.; Zhang, Y.; Zeng, X.; Qing, C.; Liu, J.; Li. L.; Zhang, H.; Bioorg. Med. Chem. Lett. 2009, 19, 5496.

21. Kalkote, U. R.; Ghorpade, S. R.; Chavan, S. P.; Ravindranathan, T.; J. Org. Chem. 2001, 66, 8277.

22. Ellman, G. L.; Courtney, K. D.; Andres, J. R. V.; Featherstone, R. M.; Biochem. Pharmacol. 1961, 7, 88.

23. Teles, A. P. C.; Takahashi, J. A.; Microbiol. Res. 2013, 168, 204.

24. Vieira, H. S.; Takahashi, J. A.; de Oliveira, A. B.; Chiari, E.; Boaventura, M. A. D.; J. Braz. Chem. Soc. 2002, 13, 151.

25. Takahashi, J. A.; Tese de Doutorado, Universidade Federal de Minas Gerais, Brasil, 1994.

26. Atanasova, M.; Stavrakov, G.; Philipova, I.; Zheleva, D.; Yordanov, N.; Doytchinova, I.; Bioorg. Med. Chem. Lett. 2015, 23, 5382.

27. Guillou, C.; Mary, A.; Renko, D. Z.; Gras, E.; Thal, C.; Bioorg. Med. Chem. Lett. 2000, 10, 637. 